\title{
Design studies for a neutrino telescope based on optical fiber hydrophones
}

\author{
E. J. Buis ${ }^{* \dagger}$ \\ TNO Technical Sciences, P.O. Box 155, 2600 AD Delft, The Netherlands \\ E-mail: ernst-jan.buis@tno.nl \\ E. Doppenberg \\ TNO Technical Sciences, P.O. Box 155, 2600 AD Delft, The Netherlands \\ E-mail: ed.doppenberg@tno.nl
}

\section{R.Lahmann}

Erlangen Centre for Astroparticle Physics, University of Erlangen, Erwin-Rommel-Str. 1, 91058

Erlangen, Germany

E-mail: robert.lahmann@physik.uni-erlangen.de

P. Toet

TNO Technical Sciences, P.O. Box 155, 2600 AD Delft, The Netherlands

E-mail: peter.toetatno.nI

\section{J. de Vreugd}

TNO Technical Sciences, P.O. Box 155, 2600 AD Delft, The Netherlands

E-mail: jan.devreugd@tno.nl

\begin{abstract}
Optical fiber hydrophones provide a promising technology for a future neutrino telescope that enables the acoustic detection of ultra-high energy neutrinos in the deep sea. These cosmic neutrinos leave a small acoustic signal that could be detected when the sensitivity of the technology is sufficiently high. In this study we have investigated in the performance of fiber optic hydrophone technology and the optimization of this technology towards the use in a neutrino telescope. We therefore measured the noise of the interrogator system and performed a sensitivity analysis of a new hydrophones sensor. A new sensor was designed, produced and characterized. It showed a dynamic range which covers a large part of the expected acoustic neutrino range. In addition, first results are shown of filtering algorithms to create a flat response of the hydrophone and to remove the resonance peaks, which are inherent to this technology.
\end{abstract}

The 34th International Cosmic Ray Conference,

30 July- 6 August, 2015

The Hague, The Netherlands

\footnotetext{
* Speaker.

† Corresponding author
} 


\section{Introduction}

Acoustic detection may provide way to observe ultra-high energy cosmic neutrinos, i.e. energies above $10^{18} \mathrm{eV}$, and their extra-galactic sources [1,2]. The expected flux of cosmic neutrinos with ultra-high energy is low, so that large scale neutrino telescopes are needed for this emerging field of astroparticle physics. Using the acoustic signals induced by a neutrino interaction in water (or ice) has the advantage that sound can travel for many kilometers with only small attenuation in the relevant frequency range. A hydrophone network that uses the sea as a detection medium may therefore be the solution to detect the ultra-high energy neutrinos.

It has been advocated that fiber optic hydrophone technology is a promising means to establish a sensitive, cost-effective and large scale sensor network [3]. In this technology several hydrophone sensors are integrated on a optical fiber. The sensors transform the acoustic pressure in to strain in the fiber. Subsequently, this strain causes a wavelength shift of the light that travels through the fiber and that is sensed using an interrogator. Hydrophones based on optical fibers, provide the required sensitivity to detect the small signals from neutrinos. At the same time, optical fibers form a cost-effective and straightforward way for the installation of a large scale network.

In this paper we discuss the system design for a fiber optic hydrophone network. We provide a flow-down from the scientific objectives to the instrument requirements. This has led to the design of a new and improved hydrophone sensor. Measurements to characterize the sensor and to show its performance will be presented. In addition the performance of the interrogator is discussed and measurements are shown, leading to an overall performance prediction of the technology.

\section{Neutrino telescope concept}

The concept of an acoustic neutrino telescope is derived from the present telescope design as implemented for instance in the Antares and KM3NeT experiment. To construct a sensitive volume in the abyss, a large number of hydrophones $(>1000)$ will be located along a large number of strings ( $>100)$. One such string will include a number of hydrophones that are read out in a multiplexed way. The string is read out using an interrogator. To be able to measure the small signals that are expected from cosmic neutrinos, the system has to exhibit the proper sensitivity. Two important aspects determine the over-al performance of the telescope. (i) The first aspect is the sensitivity of the interrogator, which is related to the minimum wavelength shift that can be measured. (ii) The second aspect is the strain sensitivity is the performance of the transducer in convert the pressure pulse into strain in the fiber.

The overall requirement in terms of the sensitivity is that the system should be able to measure signals as low a "sea state zero" at a frequency of $40 \mathrm{kHz}$. Here we use the parametrization as given by [5] that is valid in the range between 1 and $40 \mathrm{kHz}$. The power spectral density $(P)$ is shown in figure 1 together with a typical expected signal of a cosmic neutrino. Note that the noise is commonly indicated using parameter $n_{s}$, which is a continuous variable that (when discretised) denotes the deep sea state. The lowest noise level is called deep sea-state zero (DSS0) corresponds to $n_{s}=0$. At this level the lowest noise corresponds to $P_{s s 0} \approx 20 \mathrm{dBre} \mu \mathrm{Pa}^{2} / \mathrm{Hz}$ 


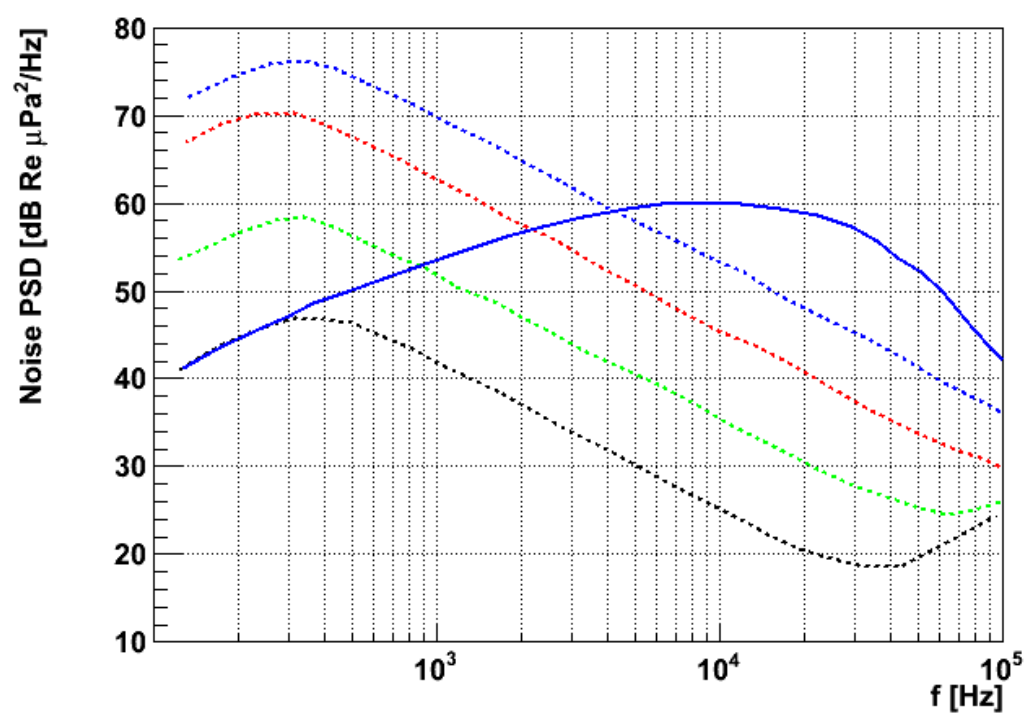

Figure 1: Sea state noise as a function of the acoustic frequency. After [4]

\section{Fiber laser hydrophones and read out}

The working principle is of the hydrophone technology is based on fiber lasers [6]. Using grating structures and a gain medium (by means of a relative high erbium doping concentration) a laser is locally inserted in the fiber. At the position of the laser a transducer is placed that converts a pressure pulse to strain on the fiber. Strain in the fiber (and hence the grating structure) causes a wavelength shift of the laser. The amount of wavelength shift $\left(\Delta \lambda_{B}\right)$ induced by the strain $(\varepsilon)$ is given [7]:

$$
\Delta \lambda_{B}=\left(1-p_{e}\right) \lambda_{B} \varepsilon
$$

where $p_{e}$ is an effective photo-elastic coefficient, which has the value $p_{e} \sim 0.22$ for fused silica.

This wavelength shift is converted to a phase shift in the interrogator using a $3 \times 3$ coupler as shown in figure 2. To determine the performance of the interrogator, the noise of the interrogator was measured in a controlled environment. For this purpose, a bare fiber (no transducer attached) was brought in a thermally and vibrational isolated environment. The resulting noise as a function of the frequency is depicted in the figure 3 (a). From the figure we read the following value for the interrogator noise: $N_{\text {system }}=-185 \mathrm{~dB}$ re $\mathrm{nm} / \sqrt{\mathrm{Hz}}$. We require that the system noise level $\left(N_{\text {system }}\right)$ is smaller than the sea state noise:

$$
N_{\text {system }}<\eta_{\text {sensor }} P_{\text {sso }}
$$

where $\eta_{\text {sensor }}$ is a conversion factor that converts the phase shift as sensed by the interrogation system to the external pressure that induced on the sensor by the sea state noise source. In figure 3 (b) we plot the required strain sensitivity as a function of the input sea state noise. We see that the required strain sensitivity on the fiber increases rapidly as the noise becomes as low as sea state zero. The conversion factor $\eta_{\text {sensor }}$ includes the conversion in the interferometer, i.e. the conversion from wavelength shift to phase shift as well as the conversion from external pressure to strain in 


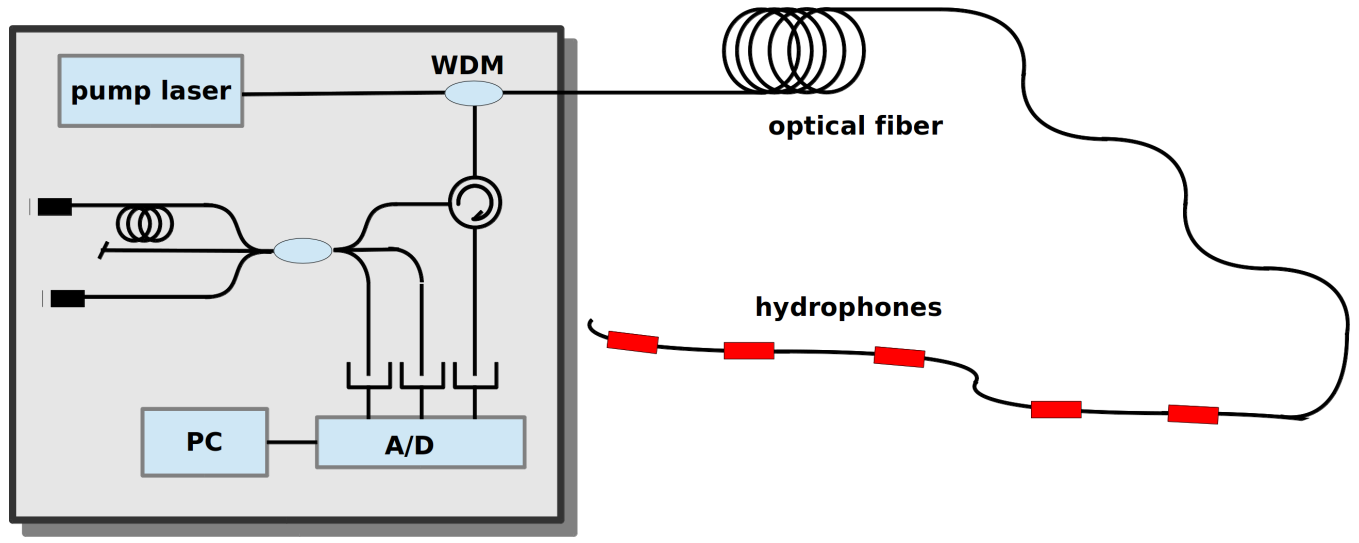

Figure 2: Fiber laser interrogator configuration using a $3 \times 3$ coupler.

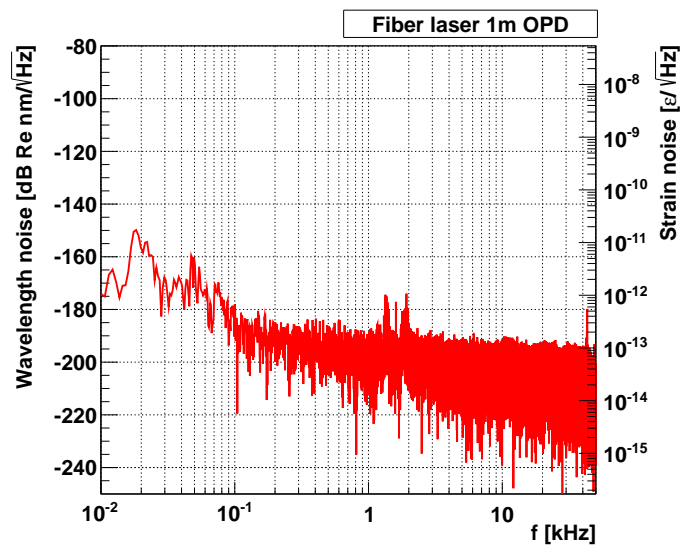

(a)

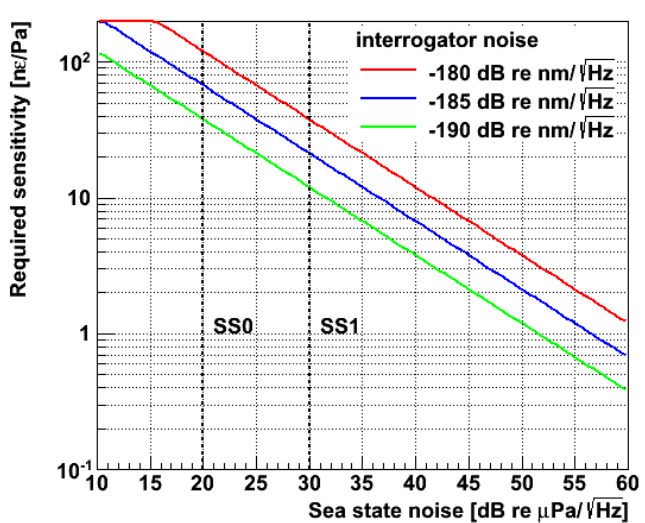

(b)

Figure 3: (a) Noise in the interrogator system. In (b) the required strain sensitivity is plotted as a function of the sea state noise for two values of interferometer noise.

the fiber. The design value for the strain sensitivity for an interferometer with a noise level of $-185 \mathrm{~dB} \mathrm{~nm} / \sqrt{\mathrm{Hz}}$ is determined to be $\eta_{\text {sensor }}=67.45 \mathrm{n \varepsilon} / \mathrm{Pa}$

\section{Sensor design and manufacturing}

A sensor was designed that met the requirements on the strain sensitivity as well the acoustic dynamical range. Various other requirements were considered as well, and in particular the requirement on the ability to produce the mechanical transducer on a large scale and in a cost-effective way. In figure 4 we plot the sensor design along with a CAD model. In addition we show the strain sensitivity as a function of the acoustic frequency. The strain sensitivity has been obtained using finite element calculations. The curve in figure 4 (b) clearly shows two resonance peaks. A series of sensors was produced of two types of material, aluminum and stainless steel respectively. The used fibers were either 80 or $125 \mu \mathrm{m}$ thickness and were glued to the sensor with a predefined pretension. 


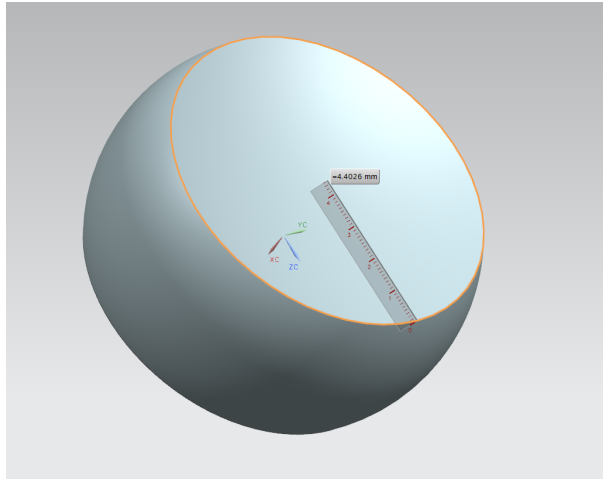

(a)

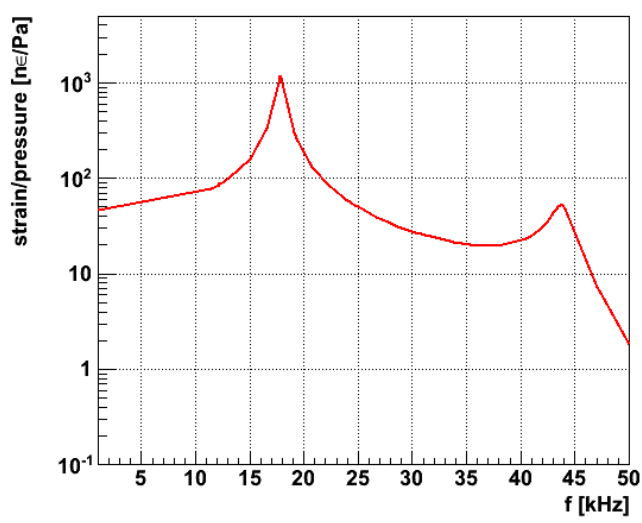

(b)

Figure 4: (a) CAD drawing of the sensor. In (b) the strain sensitivity as a function of the frequency is shown. The resonance peaks can be clearly seen.

The assembled sensor was taken to the anechoic basin facility at TNO to investigate the strain sensitivity as well as the dynamic range of the sensor. Note that the sensor was attached to an FBG, rather than a fiber laser as a dedicated fiber laser for the sensor is still under development. Although the sensor will not be sensitive enough to measure the low amplitude signals that are expected from neutrinos, the use FBG allows us to determine the strain sensitivity and to investigate in the resonance peaks of the sensor.

In figure 5 we show the measured response of the sensor. The response here is indicated using the frequency response function, which is the ratio between the hydrophone sensor under study and a reference hydrophone. In this case the reference hydrophone was a $B \& K$ hydrophone, type 8103 , that has a flat response in the frequency range of interest. Furthermore, the hydrophone was well calibrated using a dedicated pistophone. The measured response is similar to the simulated response. Again, the resonance peaks are clearly seen, although they are slightly shifted to lower frequencies compared to the peak positions as shown in figure 4 (b). The reason for this shift may be found in the glue that is used during assembly. The impact of the glue is still under investigation.

\section{Compensation of the response resonance}

The frequency response function (FRF) exhibits dominant resonances, which will filter the bipolar pulse (due to the pressure pulse) resulting in a highly un-damped response of the hydrophone. To overcome this phenomena one can mechanically redesign the sensor and introduce a mechanical damping system, which will eventually lead to unwanted decrease of the sensitivity and an unwanted increase of the overall system noise. An alternative is the use of a calibration scheme for the hydrophone response to compensate for the dominant resonances. The calibration will be performed real-time on the hydrophone response in the digital domain (thus after sampling the hydrophone response). 


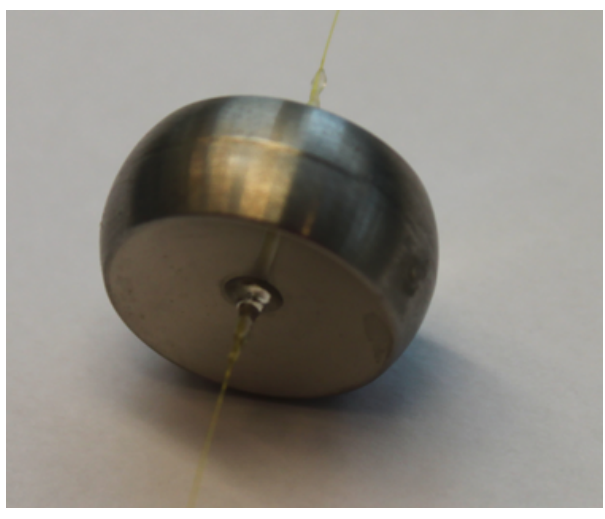

(a)

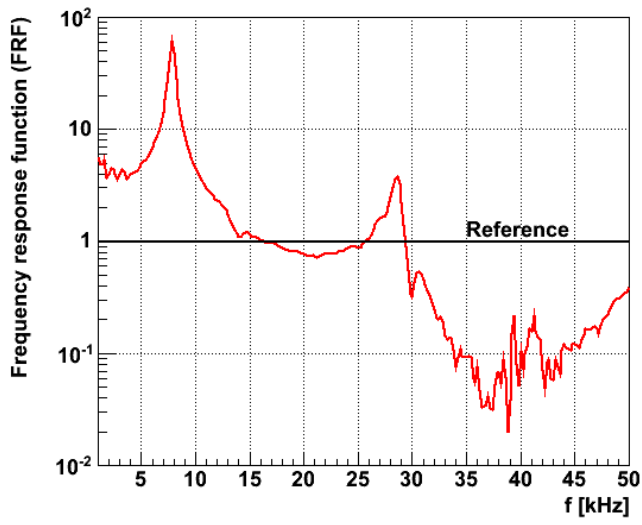

(b)

Figure 5: (a) Photograph of the hydrophone sensor. The optical fiber through the sensor can clearly be seen. In (b) the frequency response function is shown.

In general applying an inverse FRF operation in the frequency domain on the Power Spectrum of the hydrophone should do the job when a number of constraints are fulfilled:

1. The inverse of the FRF exists;

2. The inverse of the FRF is stable and causal.

However, performing the calibration in the frequency domain is relatively time consuming and rather inefficient to realize real-time response compensation. An alternative is the estimating the transfer function between the hydrophone and the reference hydrophone with a parametric model $[8,9,10]$.

A more common and natural parametric model structure is the Box-Jenkins output error model using rational transfer functions or polynomials [8]. The Box-Jenkins model structure is denoted in a general form with polynomials A, B C, D and F:

$$
A(q) \mathrm{Y}(t)=\frac{B(q)}{F(q)} \mathrm{X}(t)(t)+\frac{C(q)}{D(q)} e(t)
$$

where $e(t)$ is white noise random process with mean $\mu=0$ and variance $\sigma^{2} . q$ is the unit shift operator in the discrete (digital) domain. The selection of the order of the polynomials A, B, C, $\mathrm{D}$ and $\mathrm{F}$ will determine the model of the transfer function between the hydrophone $(\mathrm{Y}(t))$ and the reference hydrophone $(\mathrm{X}(t))$. As a proof-of-principle we have started with a simple model: Setting the polynomials $\mathrm{A}, \mathrm{C}, \mathrm{D}$ and $\mathrm{F}$ equal to 1 , gives the appropriate signal model for the transfer function. The signal model 5.1 evaluates to:

$$
\mathrm{Y}(t)=B(q) \mathrm{X}(t)+e(t)
$$

This form is the well-known Finite Impulse Response (FIR) relation between the 2 hydrophone response, which decaying to zero after a finite time is inherently stable. The inverse can be calculated and factorized in a causal and non-causal part $[9,10]$. The causal part will be used as a digital 


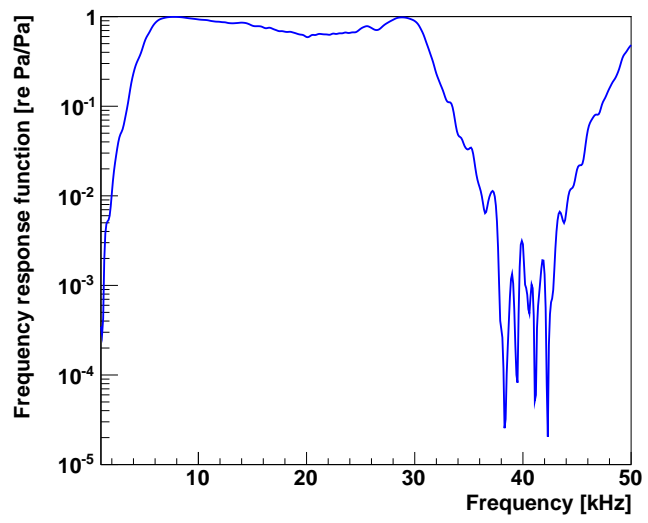

(a)

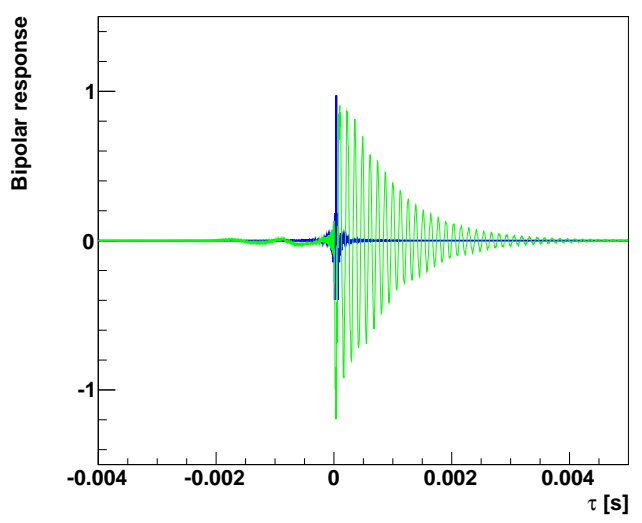

(b)

Figure 6: (a) The overall compensated Frequency response function. In (b) the 'compensated' hydrophone a response to a bipolar signal with and without compensation is shown.

filter to compensate the hydrophone response by filtering the output signal of the hydrophone realtime (It is beyond the scope of this paper to go in depth on parametric modeling and estimation).

The filtering as described above was applied to the experiments in the anechoic basin. The collected experimental data has been used to estimate the FIR model $(h(\tau))$ of the hydrophone and to calculate the inverse causal FIR model $(g(\tau))$. The compensated FRF is depicted in figure 6 (a). Clearly, compared with the FRF depicted in figure 5 (b) the dominant resonances are suppressed. However, the unwanted response near $40 \mathrm{kHz}$ remains and is partially due to the poor signal-noise-ratio of the experimental setup. To demonstrate the effect of the 'compensated' hydrophone a response to a bi-polar signal has been performed. The response is depicted in figure 6 (b). The compensated response reveals almost the 'true' form of the bi-polar pulse. The pulse is 'surrounded' by artefacts due to the poor performance near $40 \mathrm{kHz}$.

\section{Conclusions}

We have designed and produced new hydrophone sensors for the application in a neutrino telescope. The design of the sensor involved a detailed analysis of the interrogator system and its noise performance. To this end we have measured the noise of the interrogator in a controlled way. In combination of the requirement that the overall noise should be less than the sea state noise we arrived at requirements for the strain sensitivity of the sensor. A design for the sensor was made that should meet the strain sensitivity requirements, but is easy to manufacture at the same time. A finite element calculation shows that the response curve exhibits two resonance peaks. Measurements were performed in the anechoic basin at the TNO laboratories and shows the same features in the frequency response functions. A filtering technique was applied in the time domain of the data to remove real time the impact of the resonance peaks. Preliminary results show a flat response of the hydrophone up to $30 \mathrm{kHz}$. Future studies will be targeted to a even large dynamic range of the sensor as well as the further optimization of real-time filtering techniques. 


\section{References}

[1] G. A. Askaryan, Acoustic recording of neutrinos, Zemlia i Vselennaia, 1 (1979) 13

[2] J. G. Learned, Acoustic radiation by charged atomic particles in liquids: An analysis Phys. Rev. D 19 (1979) 3293

[3] E. J. Buis et al, Fibre laser hydrophones for cosmic ray particle detection, Journal of Instrumentation, 9-03 (2014) C03051

[4] R. Lahmann, Ultra-High-Energy Neutrinos and Their Acoustic Detection in the Sea, Habilitation Thesis, Friedrich-Alexander Universität Erlangen (2011)

[5] N. Kurahashi and G. Gratta, Oceanic ambient noise as a background to acoustic neutrino detection, Phys. Rev. D, (78) 9 (2008), 092001

[6] V. M. Baas et al, Fiber laser design and measurements for fiber optic hydrophones in their application for ultra-high neutrino detection, these proceedings

[7] A. D. Kersey., A Review of Recent Developments in Fiber Optic Sensor Technology, Optical Fiber Technology, 2 (1996) 291

[8] L. Ljung, System identification: theory for the user, Prentice Hall, Upper Saddle River, NJ, USA, $2^{\text {nd }}$ ed. (1999).

[9] S. Haykin, Adaptive filter theory, vol. 4th Edition, Prentice Hall (2002).

[10] A. Sayed, Fundamentals of adaptive filtering, Wiley-Interscience (2003) 